\title{
A counter-rotating core in the dwarf elliptical galaxy VCC 510
}

\author{
D. Thomas ${ }^{1,2}$, F. Brimioulle ${ }^{2,3}$, R. Bender ${ }^{2,3}$, U. Hopp ${ }^{2,3}$, L. Greggio ${ }^{4}$, C. Maraston ${ }^{1,2}$, and R. P. Saglia ${ }^{2}$ \\ ${ }^{1}$ University of Oxford, Denys Wilkinson Building, Keble Road, Oxford, OX1 3RH, UK \\ e-mail: dthomas@astro.ox.ac.uk \\ 2 Max-Planck-Institut für extraterrestrische Physik, Giessenbachstraße, 85748 Garching, Germany \\ 3 Universitäts-Sternwarte München, Scheinerstr. 1, 81679 München, Germany \\ 4 INAF, Osservatorio Astronomico di Padova, vicolo dell'Osservatorio 5, 35122 Padova, Italy
}

Received 27 October 2005 / Accepted 13 November 2005

\section{ABSTRACT}

Aims. We present optical long-slit spectra of the Virgo dwarf elliptical galaxy VCC 510 at high spectral $\left(\sigma \sim 30 \mathrm{~km} \mathrm{~s}^{-1}\right)$ and spatial resolution. The principal aim is to unravel its kinematical and stellar population properties.

Methods. Heliocentric velocities and velocity dispersions as functions of galaxy radius are derived by deconvolving line-of-sight velocity distributions. The luminosity-weighted stellar population parameters age and element abundances are obtained by comparison of Lick absorption-line indices with stellar population models.

Results. A maximum rotation $v_{\text {rot }}=8 \pm 2.5 \mathrm{~km} \mathrm{~s}^{-1}$ inside half the effective radius $\left(r_{\mathrm{e}} \approx 20^{\prime \prime}\right)$ and a mean, radially flat velocity dispersion $\sigma=44 \pm 5 \mathrm{~km} \mathrm{~s}^{-1}$ are measured. The core extending over the inner $2^{\prime \prime}(\sim 140 \mathrm{pc})$ is found to rotate in the opposite sense with $v_{\text {rot }}^{\text {core }} \approx-1 / 2 v_{\text {rot }}$. VCC $510\left(M_{B} \sim-15.7\right)$ is therefore by far the faintest and smallest galaxy with a counter-rotating core known. From the main body rotation and the velocity dispersion profile we deduce that VCC 510 is anisotropic and clearly not entirely supported by rotation. We derive an old luminosity-weighted age $(10 \pm 3 \mathrm{Gyr})$ and sub-solar metallicity $([\mathrm{Z} / \mathrm{H}]=-0.4 \pm 0.1)$ inside the effective radius. There is tentative evidence that the counter-rotating core might be younger and less $\alpha / F e$ enhanced. From the stellar population parameters we obtain a total stellar massto-light ratio of $\sim 3.6\left(M / L_{B}\right)_{\odot}$ which is significantly lower than a rough dynamical estimate obtained from the kinematics through the virial theorem $(\sim 15)$. This discrepancy hints toward the possible presence of dark matter in the centre of VCC 510.

Conclusions. We discuss the origin of the counter-rotating core and exclude fly-by encounters as a viable possibility. Gas accretion or galaxy merging provide more likely explanations. VCC 510 is therefore the direct observational evidence that such processes do occur in cluster satellite galaxies on dwarf galaxy scales.

Key words. galaxies: dwarf - galaxies: kinematics and dynamics - galaxies: formation - galaxies: interactions - galaxies: stellar content

\section{Introduction}

Counter-rotating cores are found in a considerable fraction of giant early-type galaxies (e.g., Bender 1988; Franx \& Illingworth 1988; Jedrzejewski \& Schechter 1988; Bender \& Surma 1992; Bertin et al. 1994; Graham et al. 1998; Mehlert et al. 1998; Wernli et al. 2002; Emsellem et al. 2004). Objects with this kinematic peculiarity constitute a subclass of an even more common group of galaxies hosting so-called kinematically decoupled components (KDCs). First discovered by Efstathiou et al. (1982), the latter are cores that rotate at different speed from the main body of the galaxy. Their formation, and in particular the formation of counter-rotating cores, is best understood in a merging scenario, hence their presence is considered to be very direct evidence that merging plays a rôle in the formation of giant early-type galaxies (Kormendy 1984; Balcells \& Quinn 1990).
Since its discovery in the late 80 's, this interesting kinematical feature has been restricted to massive, luminous galaxies. For our understanding of galaxy formation, it is certainly of great interest to know whether this limitation is of pure observational nature and whether merger remnants with these kinematical fingerprints exist also on smaller galaxy scales. A first hint that this might be the case comes from recent observations by Geha et al. (2005), who found a counter-rotating core in the low-luminosity elliptical NGC 770, being the faintest galaxy $\left(M_{B} \sim-18\right)$ with this kinematical feature known at that time (see also Prugniel et al. 2005).

First evidence that the presence of kinematical peculiarities in galaxy centres extends even further down to dwarf galaxy scales is provided by De Rijcke et al. (2004). The authors have detected kinematic decoupling in the centres of two dwarf elliptical galaxies, where in both cases a pronounced bump in the rotation velocity profile at a radius of $1^{\prime \prime}(\sim 200 \mathrm{pc})$ is found. The cores of these objects rotate in the same sense and with 


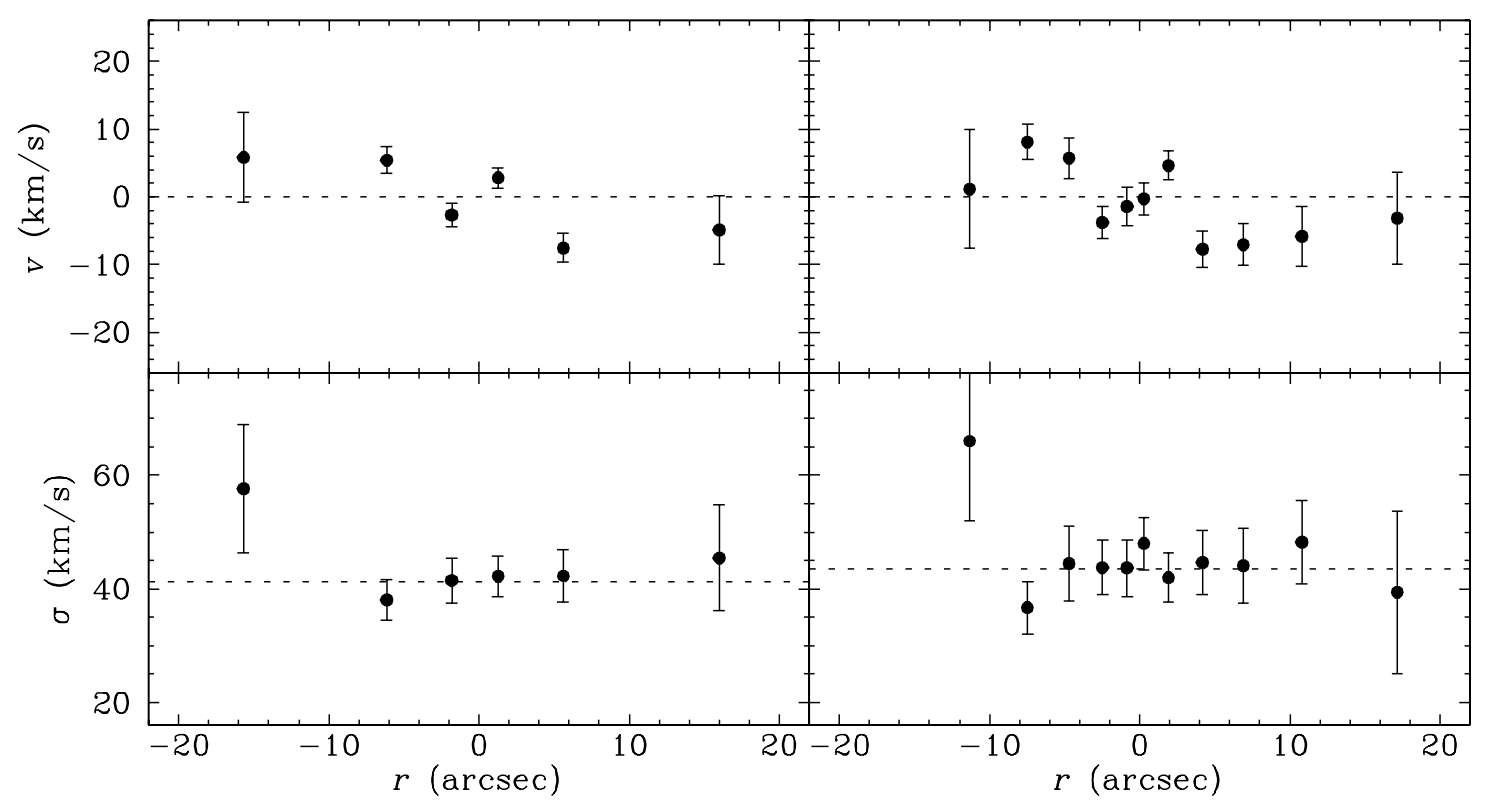

Fig. 1. Heliocentric velocity $v$ (mean $v$ subtracted) and velocity dispersion as functions of radius. Coarse and fine binnings are shown by the left and right panels, respectively. 1" corresponds roughly to $70 \mathrm{pc}$ adopting $14.6 \mathrm{Mpc}$ for the distance of the Virgo cluster (Freedman et al. 2001).

similar velocities as the main body. De Rijcke et al. (2004) argue that this feature is best understood in a harassment scenario and is most likely caused by fly-by interaction with the nearby massive galaxy.

In this paper we report on the detection of a counterrotating core in a dwarf elliptical galaxy. We present the kinematics of VCC 510, a nucleated dwarf elliptical of the Virgo cluster (Binggeli et al. 1985; Binggeli \& Cameron 1991, 1993) with moderate flattening ( $\epsilon=0.18$ ), a total blue luminosity of $M_{B} \sim-15.7$, and an effective radius of $r_{\mathrm{e}}=20^{\prime \prime} \sim 1.4 \mathrm{kpc}$. We assume $14.6 \pm 0.04 \mathrm{Mpc}$ for the distance to the Virgo cluster (Freedman et al. 2001). Being more than two magnitudes fainter than NGC 770, VCC 510 is by far the smallest galaxy known with a counter-rotating core.

\section{Observations and data analysis}

The observations were performed at the $3.5 \mathrm{~m}$ telescope (TWIN spectrograph) of the Calar Alto Observatory (Spain) in April 2003. A long-slit (1.8" width) was placed at the centre of VCC 510 aligned along the major axis, yielding a wavelength coverage $4650 \lesssim \lambda / \AA \lesssim 5600$ at relatively high instrumental resolution $\left(\sigma_{\text {inst }} \sim 30 \mathrm{~km} \mathrm{~s}^{-1}\right)$. A total exposure time of $5.5 \mathrm{~h}$ yielded a signal-to-noise ratio $\left(\AA^{-1}\right)$ of 30 for fine and 40 for coarse radial binning (see Fig. 1) inside $r_{\mathrm{e}}$, with a seeing of about $2^{\prime \prime}$. We also observed 20 standard stars serving as templates for both the kinematic analysis and the calibration of Lick absorption-line measurements (see below). VCC 510 is one of the 35 dwarf early-type galaxies mostly from the Virgo cluster we have observed over the past 10 years. The complete sample will be presented in a future paper, full details about data reduction will be given there. In the following we briefly summarise the essentials.

The standard CCD data reduction (bias \& dark subtraction, flat fielding, wavelength calibration, and sky subtraction) was carried out under the image processing package MIDAS provided by ESO. Line-of-sight-velocity-distributions were determined by using the Fourier-Correlation-Quotient (FCQ) method from Bender (1990), with which we derived heliocentric velocities and velocity dispersions (Bender et al. 1994). We measured the Lick absorption-line indices $\mathrm{H} \beta, \mathrm{Mg} b, \mathrm{Fe} 5270$, and Fe5335 (Worthey et al. 1994). For this purpose, the spectra were degraded to Lick resolution, and the intrinsic velocity broadening, even though almost negligible in case of dwarf galaxies, was taken into account. The measurements are calibrated onto the Lick system by means of the 20 Lick standard stars observed. For both kinematics and line indices, statistical errors were derived from Monte Carlo simulations.

\section{Results}

\subsection{Kinematics}

The rotation curve and the radial velocity dispersion profile of VCC 510 are shown in Fig. 1 for coarse (left-hand panel) and fine (right-hand panel) radial binning. In both cases, but in particular for the fine binning, the counter-rotating core is clearly visible. The core region inside $2^{\prime \prime}(\sim 140 \mathrm{pc})$ is rotating with about $4 \mathrm{~km} \mathrm{~s}^{-1}$ in a sense opposite to the main body rotation. We are confident that this feature is real, as it is robust against variations of the radial binning as shown in Fig. 1. Note also that the rotation velocity of the core should be regarded as a lower limit, because the moderate seeing of the present observations contributes to a dilution of the signal. The maximum rotation velocity of the galaxy (at $\sim 6^{\prime \prime}$ ) is $v_{\text {rot }}=8 \pm 2.5 \mathrm{~km} \mathrm{~s}^{-1}$.

The velocity dispersion of VCC 510 is $\sigma=44 \pm 5 \mathrm{~km} \mathrm{~s}^{-1}$ and reveals no radial dependence inside $r_{\mathrm{e}}$, which is a typical characteristic for dwarf elliptical galaxies (Bender \& Nieto 1990; Bender et al. 1991; Geha et al. 2003). The resulting relatively low $v / \sigma=0.18 \pm 0.06$ at the ellipticity $\epsilon=0.18$ shows that VCC 510 is supported by anisotropic velocity dispersion 

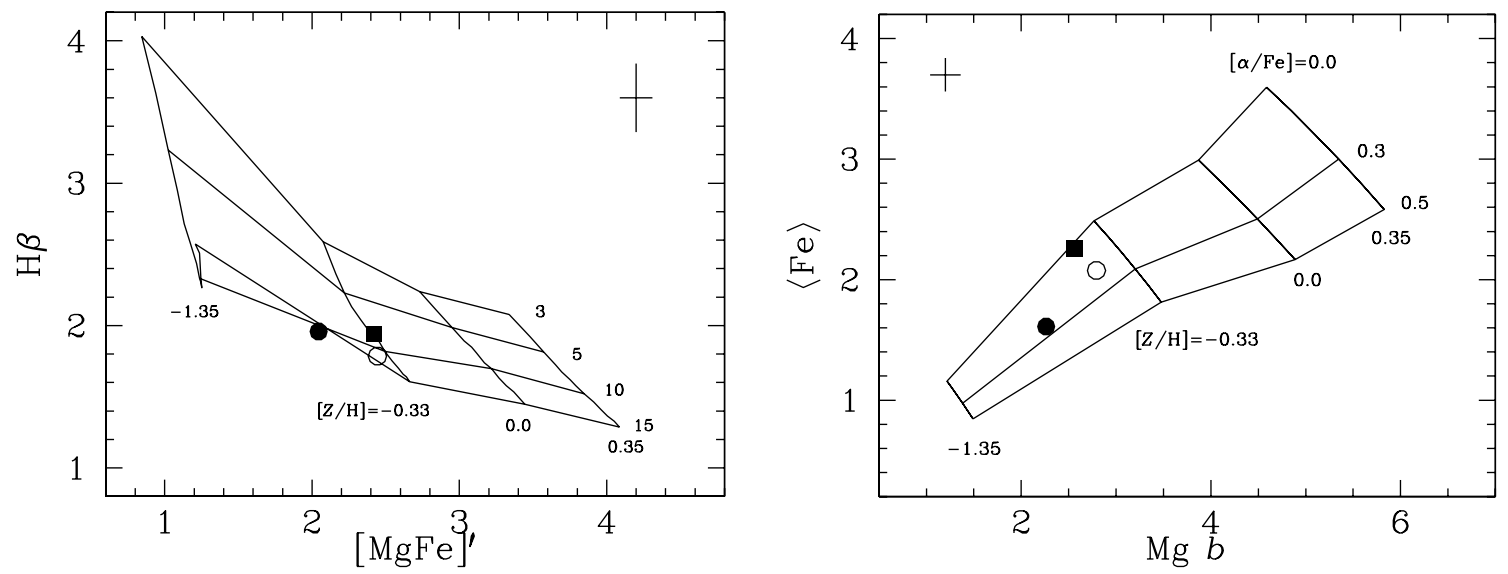

Fig. 2. Lick absorption-line indices for three radial bins: very centre (filled circle), at $r=2^{\prime \prime}$ (filled square, radius of the counter-rotating core), and at $r=5^{\prime \prime}$ (open circle). Stellar population models (Thomas et al. 2003) for various ages, metallicities, and $\alpha / \mathrm{Fe}$ ratios (see labels) are over-plotted. In the left panel $\alpha / \mathrm{Fe}$ is fixed to solar, in the right panel age is fixed to 10 Gyr. For index definitions see Thomas et al. (2003).

Table 1. Kinematical parameters of VCC 510.

\begin{tabular}{|c|c|c|c|c|c|c|c|c|c|}
\hline $\begin{array}{c}M_{B} \\
(\mathrm{mag})\end{array}$ & $\begin{array}{c}r_{\mathrm{e}} \\
(\operatorname{arcsec})\end{array}$ & $\epsilon$ & $\begin{array}{c}v_{\text {hel }} \\
\left(\mathrm{km} \mathrm{s}^{-1}\right)\end{array}$ & $\begin{array}{c}v_{\text {rot }} \\
\left(\mathrm{km} \mathrm{s}^{-1}\right)\end{array}$ & $\begin{array}{c}v_{\mathrm{rot}}^{\text {core }} \\
\left(\mathrm{km} \mathrm{s}^{-1}\right)\end{array}$ & $\begin{array}{c}\sigma \\
\left(\mathrm{km} \mathrm{s}^{-1}\right)\end{array}$ & $v / \sigma$ & $\begin{array}{c}M_{\mathrm{dyn}} \\
\left(10^{9} M_{\odot}\right)\end{array}$ & $\begin{array}{c}\left(M / L_{B}\right)_{\mathrm{dyn}} \\
\left(M / L_{B}\right)_{\odot}\end{array}$ \\
\hline-15.7 & 20 & 0.18 & $858 \pm 1$ & $8.0 \pm 2.5$ & $\sim-1 / 2 v_{\text {rot }}$ & $44 \pm 5$ & $0.18 \pm 0.06$ & $4.5 \pm 0.5$ & $15 \pm 2$ \\
\hline
\end{tabular}

(Binney 2005; Burkert \& Naab 2005). A significant increase of $v_{\text {rot }}$ beyond $r_{\mathrm{e}}$, as found by Simien \& Prugniel (2002) for NGC 205, cannot be excluded, but seems unlikely. Figure 1 indicates even a slight decrease of the rotation velocity at the outermost point.

Finally, following the recipe of Binney \& Tremaine (1987), we obtain the virial mass $M_{\mathrm{vir}}=4.5 \pm 0.5 \times 10^{9} M_{\odot}$, which can be considered a rough estimate of the total dynamical mass. This leads to a relatively high dynamical $B$-band mass-to-light ratio of $15 \pm 2\left(M / L_{B}\right)_{\odot}$. The results are summarised in Table 1 .

\subsection{Stellar populations}

In Fig. 2 we show the Lick absorption-line indices measured for three radial bins and confront them with stellar population models of various ages, metallicities, and $\alpha / \mathrm{Fe}$ ratios as indicated by the labels (Thomas et al. 2003). Using the method explained in detail in Thomas et al. (2005), we derive the following global (within $5^{\prime \prime}$ ) luminosity-weighted stellar population parameters: age $t \approx 10.3 \pm 2.8 \mathrm{Gyr}$, metallicity $[\mathrm{Z} / \mathrm{H}]=$ $-0.4 \pm 0.13$, and abundance ratio $[\alpha / \mathrm{Fe}]=0.07 \pm 0.08 \mathrm{dex}$. Most importantly in the context of this letter, the counterrotating core does not show any clear peculiarity in the stellar population properties within the measurement errors, in particular no obvious sign of recent star formation. This seems indeed to be a quite common characteristic for counter-rotating cores in elliptical galaxies (Surma \& Bender 1995; Mehlert et al. 1998; Davies et al. 2001; Morelli et al. 2004).

However, a closer look at the figure suggests that the stellar populations at the radius of the counter-rotating core (square) might be somewhat younger by about $2 \mathrm{Gyr}$, and less alpha/Fe enhanced by $\sim 0.2$ dex. But, given the measurement errors, this result can be considered indicative at best. Assuming the core contributes about 10-30 per cent in mass (enough to produce counter-rotating cores, see Surma \& Bender 1995), this slight rejuvenation in the core region would imply the presence of a young component with age $t \sim 4-6 \mathrm{Gyr}$, corresponding to formation redshifts of $z \sim 0.5-0.8$.

Based on the stellar population models of Maraston (2005), the global mean age and metallicity derived above translate into the stellar mass-to-light ratio $\left(M / L_{B}\right)_{*}=3.6 \pm 0.7\left(M / L_{B}\right)_{\odot}$ assuming a Kroupa initial mass function (Salpeter increases the $\mathrm{M} / \mathrm{L}$ by a factor $\sim 1.6$ ). This value is significantly smaller than the dynamical estimate presented above. Unless the simple recipe used here over-estimates the mass by more than a factor 3, this indicates that dark matter might be present in the centre of VCC 510. Sophisticated modelling based on more observational data is certainly needed for a detailed assessment.

\section{Discussion and conclusion}

Given the evidence presented here, there is no doubt that VCC 510 contains a counter-rotating core. The challenge is now to understand (and model) the origin of this kinematic peculiarity. It is widely accepted that galaxy mergers are the key process to form kinematically decoupled cores in massive galaxies (see Introduction and references therein). Is this scenario valid also for dwarfs?

De Rijcke et al. (2004) argue that mergers between small galaxies are generally unlikely. In a dense environment velocities are too high, and in looser environments densities are too low. They suggest an alternative explanation, according to which the internal kinematics of a dwarf galaxy are modified by fly-by encounters with a massive companion. Developing 
a simple analytical description, they estimate that this harassment process can produce a kinematic decoupling in the centres of dwarf ellipticals, as long as impact parameters of the interaction are not larger than $\sim 20 \mathrm{kpc}$.

While it might indeed be plausible that fly-by interaction affects internal kinematics leading to perturbations of the inner core as found in De Rijcke et al. (2004), it seems highly unlikely that the sense of rotation can be completely reversed by this mechanism (González-García et al. 2005). Adapting Eq. (7) of De Rijcke et al. (2004), we find no sensible combination of parameters (velocity dispersion in Virgo $\sim 600 \mathrm{~km} \mathrm{~s}^{-1}$; companion mass around $10^{11} M_{\odot}$; impact parameter $\gtrsim 20 \mathrm{kpc}$ ) that would affect the rotation velocity significantly enough to explain the present observations. Geha et al. (2005) come to the same conclusion for the low-mass elliptical NGC 770.

A galaxy merger or gas accretion are the alternatives. According to the commonly accepted scenario, a dissipationless, unequal-mass merger can produce a kinematically decoupled core (Kormendy 1984; Balcells \& Quinn 1990), under the premise that the nucleus of the accreted galaxy is denser. This is plausible for giants, as surface brightness indeed increases with decreasing galaxy mass, but is a bit problematic for dwarfs, where the opposite relation is observed (e.g., Kormendy 1985). More compelling might be a dissipational merger or gas accretion accompanied by star formation. The counter-rotating core of NGC 4365, for instance, has formed most probably through such a process, as it is found to have disc-like structure, low kinematic temperatures and a significantly flattened density distribution (Surma \& Bender 1995).

The latter scenario gets support from the indication that the counter-rotating core might have experienced recent star formation as discussed in Sect. 3.2. In this case, the core should exhibit a disc-like structure. Clearly, photometry and spectroscopy at much higher spatial resolution than presented here are needed to verify the presence of such a cold component in the centre.

Mergers between dwarfs, on the other hand, are quite unlikely in clusters given their low cross-section and the high relative velocities. Based on $N$-body simulations by Makino \& Hut (1997) and adopting the dwarf galaxy surface density of Virgo from Phillipps et al. (1998), we estimate that only 1 out of 400 dwarf ellipticals in the Virgo cluster should have experienced a merger over a Hubble time.

VCC 510 is the direct evidence that gas accretion or mergers between satellites do occur on dwarf galaxy scales. They might be important for galaxy formation modelling (Menci et al. 2002), and it needs to be assessed in future how common this phenomenon really is.

Acknowledgements. We are grateful to the Calar Alto staff for the observations, to J. Binney, R. Davies, and S. Khochfar for interesting discussions, and to E. Emsellem for the very quick and careful report. DT acknowledges financial support by grant BMBF-LPD 9901/8-111 of the Deutsche Akademie der Naturforscher Leopoldina.

\section{References}

Balcells, M., \& Quinn, P. J. 1990, ApJ, 361, 381

Bender, R. 1988, A\&A, 202, L5

Bender, R. 1990, A\&A, 229, 441

Bender, R., \& Nieto, J.-L. 1990, A\&A, 239, 97

Bender, R., \& Surma, P. 1992, A\&A, 258, 250

Bender, R., Paquet, A., \& Nieto, J.-L. 1991, A\&A, 246, 349

Bender, R., Saglia, R. P., \& Gerhard, O. 1994, MNRAS, 269, 785

Bertin, G., Bertola, F., Buson, L. M., et al. 1994, A\&A, 292, 381

Binggeli, B., \& Cameron, L. M. 1991, A\&A, 252, 27

Binggeli, B., \& Cameron, L. M. 1993, A\&AS, 98, 297

Binggeli, B., Sandage, A., \& Tammann, G. A. 1985, AJ, 90, 1681

Binney, J. 2005, MNRAS, 363, 937

Binney, J., \& Tremaine, S. 1987, Galactic Dynamics (New York: Princeton University Press)

Burkert, A., \& Naab, T. 2005, MNRAS, 363, 597

Davies, R. L., et al. 2001, ApJ, 548, L33

De Rijcke, S., Dejonghe, H., Zeilinger, W. W., \& Hau, G. K. T. 2004, A\&A, 426, 53

Efstathiou, G., Ellis, R. S., \& Carter, D. 1982, MNRAS, 201, 975

Emsellem, E., Cappellari, M., Peletier, R. F., et al. 2004, MNRAS, 352,721

Franx, M., \& Illingworth, G. D. 1988, ApJ, 327, 55

Freedman, W. L., Madore, B., Gibson, B., et al. 2001, ApJ, 553, 47

Geha, M., Guhathakurta, P., \& van der Marel, R. P. 2003, AJ, 126, 1794

Geha, M., Guhathakurta, P., \& van der Marel, R. P. 2005, AJ, 129, 2617

González-García, A. C., Aguerri, J. A. L., \& Balcells, M. 2005, A\&A, in press [arXiv: astro-ph/0508610]

Graham, A. W., Colless, M. M., Busarello, G., Zaggia, S., \& Longo, G. 1998, A\&AS, 133, 325

Jedrzejewski, R., \& Schechter, P. L. 1988, ApJ, 330, L87

Kormendy, J. 1984, ApJ, 287, 577

Kormendy, J. 1985, ApJ, 295, 73

Makino, J., \& Hut, P. 1997, ApJ, 481, 83

Maraston, C. 2005, MNRAS, 362, 799

Mehlert, D., Saglia, R. P., Bender, R., \& Wegner, G. 1998, A\&A, 332, 33

Menci, N., Cavaliere, A., Fontana, A., Giallongo, E., \& Poli, F. 2002, ApJ, 575, 18

Morelli, L., Halliday, C., Corsini, E. M., et al. 2004, MNRAS, 354, 753

Phillipps, S., Parker, Q. A., Schwartzenberg, J. M., \& Jones, J. B. 1998, ApJ, 493, L59

Prugniel, P., Chilingarian, I., Sil'chenko, O., \& Afanasiev, V. 2005, in Near-field cosmology with dwarf elliptical galaxies, ed. B. Binggeli, IAU Coll., 198, in press

[arXiv: astro-ph/0510398]

Simien, F., \& Prugniel, P. 2002, A\&A, 384, 371

Surma, P., \& Bender, R. 1995, A\&A, 298, 405

Thomas, D., Maraston, C., \& Bender, R. 2003, MNRAS, 339, 897

Thomas, D., Maraston, C., Bender, R., \& Mendes de Oliveira, C. 2005, ApJ, 621, 673

Wernli, F., Emsellem, E., \& Copin, Y. 2002, A\&A, 396, 73

Worthey, G., Faber, S. M., González, J. J., \& Burstein, D. 1994, ApJS, 94, 687 